\title{
Applicability of near infrared sorting in the minerals industry
}

M.R. Robben, H. Knapp and H. Wotruba

Unit of Mineral Processing, RWTH Aachen University, Lochnerstrasse 4-20, 52074 Aachen, Germany. E-mail: robben@amr.rwth-aachen.de

\section{Introduction}

$\mathrm{t}$ is not possible to eliminate near infrared (NIR) spectroscopy as an analytical technique from our modern daily life. Flinn describes this very comprehensively and includes various application examples of NIR spectroscopy in his article titled "An average day (or how near infrared affects daily life)" in 2005. Applications of NIR spectroscopy in the minerals processing industry were not mentioned in the article in contrast to, for example, applications in the recycling industry. The technique is not yet widely applied in the minerals industry though, as in other industries, its use is expanding rapidly.

The following article offers a very brief overview of the applicability of NIR spectroscopy in the minerals industry with specific attention for the latest research on NIR sorting. Since 2008, the Unit of Mineral Processing (AMR) (RWTH Aachen University) has conducted research on the feasibility of NIR sorting. This research has unfolded the potential and led to the implementation of the first NIR sorters in the minerals industry. ${ }^{2}$

\section{NIR spectroscopy in the minerals industry}

With NIR spectroscopy it is possible to identify various minerals and a major amount of geological material, obtaining information on the chemical composition and the context in which the minerals occur. ${ }^{3}$ The minerals industry offers various fields of application for NIR spectroscopy. The physics of NIR spectroscopy for geological material are the same whether applied to planetary surfaces or rock moving on a conveyor belt; this makes it easy to transfer the knowledge between different application fields.

NIR spectroscopy can be implemented as an off-line laboratory analysis as well as for on-line process control. In both cases the well-known advantages (relatively lowcost, non-destructive, easy sample preparation, short analysis time) are good reasons for using NIR spectroscopy in the minerals

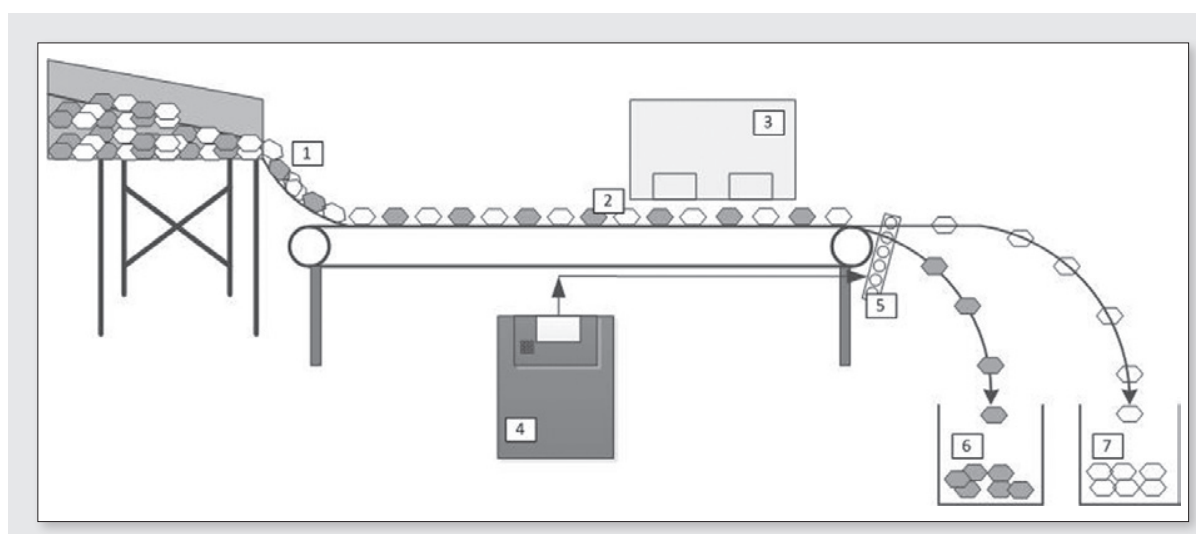

Figure 1. Functional principle of a typical sensor-based sorter. (1) Material conditioning, (2) Material presentation, (3) Material sensing, (4) Data processing, (5) Material separation, (6) Accepted fraction, (7) Rejected fraction

industry. Currently available methods [X-ray diffraction, mineral liberation analysis and scanning electron microscopy (QEMSCAN)] for the identification of mineralogical composition are time-consuming and relatively expensive. NIR spectroscopy allows the execution of mineralogy measurements at a frequency that otherwise would not be practical or affordable. On-line analysis enhances process planning and efficiency because the operator gets real-time quality information and is thus able to react to short time changes in material properties. For online analyses, the NIR system is mostly mounted above the conveyor belt. NIR on-line measurements are already implemented in the minerals industry to measure, for example, moisture content and the quality of limestone and cement (e.g. the $\mathrm{MgO}$ content).

NIR spectroscopy has been widely used in remote sensing for 40 years. Mostly a combination of airborne, space-born and ground portable systems is used for exploration purposes. Mineral components in the surface, as well as vegetation stressed by metals in the substrate can be identified. ${ }^{4}$ Besides the application of NIR spectroscopy for characterising and mapping hydrothermal systems and deposits, NIR spectroscopy can also be used for many other mining applications such as, for example, the detection of acid mine drainage.

The latest application field in the minerals industry is sorting and NIR spectroscopy can be applied as a surface sensing technique in a sensor-based sorter. Sensorbased sorting (SBS) is an automatic sorting technique in which particles in a material flow are singularly detected and evaluated by a sensor and then rejected by an amplified mechanical, hydraulic or pneumatic process.

\section{Sensor-based sorting}

Figure 1 shows a typical sensor-based sorting system and illustrates the fundamental principle and main sub-processes. The main sub-processes are material conditioning (1), material presentation (2), material sensing (3), data processing (4) and separation (5). SBS is based on differences in physical properties which can be detected by a sensor. Various different sensor technologies (optical, electromagnetic, Dual Energy X-Ray transmission, X-ray fluorescence, neutron activation and NIR spectroscopy) are applicable. After detection and qualification of one or more of those properties, all the information is evaluated to decide whether the particle is discharged and the separation system is actuated or not. The 
resulting fractions are called "accepted fraction" (6) and "rejection fraction" (7) with one of them being the concentrate (product) and one of them being the tailings (waste). The fraction with the smaller mass flow is usually the rejected fraction to save energy at the rejection process. ${ }^{5}$ The separation is usually achieved by a pneumatic blast produced by an array of high-speed air valves.

SBS is on its way to becoming a conventional process technique in the minerals industry. It has been successfully implemented in more than a thousand cases and is used to fulfil various tasks. ${ }^{5}$ The basic aim of SBS is to improve the grade of the ore stream flowing into subsequent mineral processing operations. This aim is achieved by waste reduction (remove barren) and/or removal of material that causes challenges in the conventional process, like acid-consuming carbonate. SBS can, for example, be implemented in the processes as a pre-concentration step (waste removal at an early stage) for coarse particles (up to $300 \mathrm{~mm}$ ), for ore type diversion into different processing streams and waste dump retreatment. If effort is spent on calibration, sensor-based sorters can be used for online analysis. They can deliver online data on particle size distribution, grade and, in the case of NIR, mineralogy. SBS is a clean and green processing technique needing no or little water and little power compared to other pre-concentration techniques.

\section{NIR sorting}

As mentioned before, it is possible to transfer knowledge from other application fields to sorting applications. However, sorting in the minerals industry has special requirements, especially because of the material properties and the conditions in the processing plants, such as dust levels and detection speed. The feed of the sorter has a relatively high variability in, for example, size, mineral content and surface moisture. A belt speed of $3 \mathrm{~ms}^{-1}$ and a throughput of 300 tonnes per hour are common figures. Although NIR sorting is technically viable down to $6 \mathrm{~mm}$, it is normally applied in ranges larger than $30 \mathrm{~mm}$. The maximum particle size is $300 \mathrm{~mm}$ due to mechanical restriction of the physical separation process. $^{14}$

\section{Functional principle of NIR sorting}

In the case of NIR sorting, the detected physical property is the reflectance of NIR

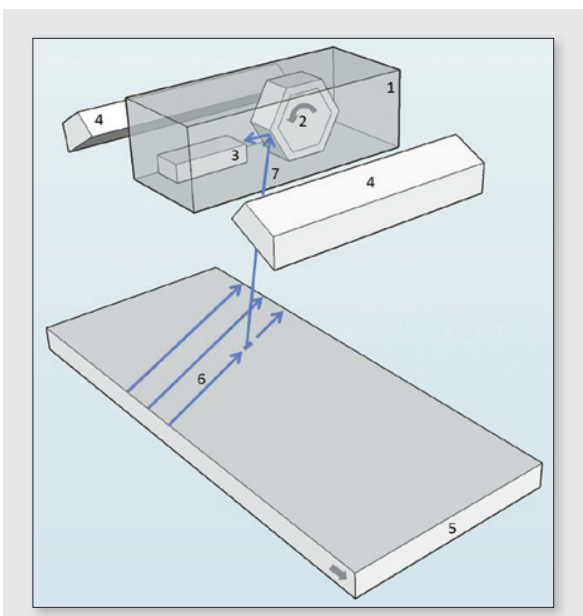

Figure 2. Technical set up of a line scan sorter. 1) NIR sensor system, 2) Rotating polygon mirror, 3) Detector, 4) NIR light source, 5) Conveyor belt 6) Path of detecting point, 7) Reflected light beam.

radiation which is emitted by the source above the belt. The diffusely reflected radiation is directed to the detector unit. If spectral differences can be recovered, then sorting is theoretically possible. The current state-of-the-art is the measurement of 640,000 NIR spectra per second per metre conveyor belt. The most common setup applied in NIR sorting is a line scan camera. The technical setup is mounted above the belt and consists of a detector and a perpendicular (in relation to the belt's moving axis) rotating polygon mirror (Figure 2). This mirror moves and reflects the point signals into the spectrometer allowing the point scanner to scan the whole belt width. On a moving belt, the two movements of the scanning point and the belts velocity overlap and form an angular scanning pattern on the belt.

The collected data consists of a hypercube with three coordinates $(x, y, \lambda)$ where $\lambda$ is the spectral response. It is possible to combine the measurement with object detection software. Data enhancement typically involves normalisation and classification algorithms. The required time and complexity of the classification procedure can be reduced by limiting the data processing to spectra relevant areas.

\section{Potential of NIR sorting}

NIR sorting is widely-applied in the recycling and food industries though acceptance of this technology in the minerals processing industry has been relatively low. Comprehensive test work on ores, including ores inseparable with traditional SBS techniques, shows encouraging results with NIR sorting. ${ }^{2,6}$ The results for sorting barite, fluorite, calcite, marble, talc, borate, zinc and iron are very encouraging. If the major components do not show specific absorption features, minor components like alteration minerals can be used as markers for the sorting task as indirect sorting criteria. ${ }^{6}$ In 2011, the first NIR sorters found their applications in the minerals industry.

Homogeneous material is preferable for the sorting. The spectral response of heterogeneous material, which is normally the case in mining, is much more complicated; wavelength shifts, overtones, overlapping of the absorption features etc. and mixed spectra occur. Mixed spectra are caused by diluted minerals, contact zones of different rock types (with different mineral contents), dust coatings and the presence of water (hydroscopic and free pore water). All this spectral variation needs to be taken into account during the design of the sorting algorithms. Other factors like shape and temperature also cause spectral variations but if the spectral variations are smaller than the difference caused by chemical variance, sorting can take place. ${ }^{6}$

\section{Application example}

The application example, NIR sorting of talc, was published by Robben et al. in 2012. ${ }^{2}$ It is a very good example of the potential of NIR sorting in the minerals industry, especially because no other sensor-based sorting method was applicable for the problem. With NIR sorting, the bright coloured carbonate can be distinguished from talc which is an advantage over colour sorting.

As a natural ore, talc is always found in combination with other minerals like chlorite, a chemically and structurally similar ore, carbonates and quartz. These associated minerals can bring undesirable loss of performance in specific applications, for example ceramics and polymers. Therefore, the AMR has conducted a feasibility study to test the applicability of separating talc from its main contaminants based on the spectral response in the NIR region.

\section{Trials on a desktop spectrometer}

Samples were analysed using the external sphere module of a desktop NIR spectrometer (Tensor 27, Bruker Optics Inc.) in the 


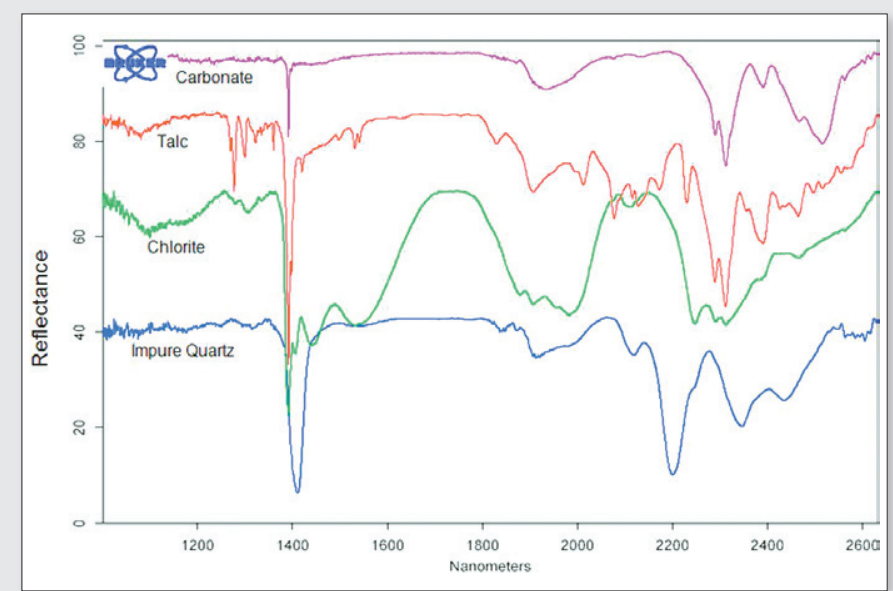

Figure 3. Representative spectra of four different rock types (baselinecorrected and offset on the vertical axis).

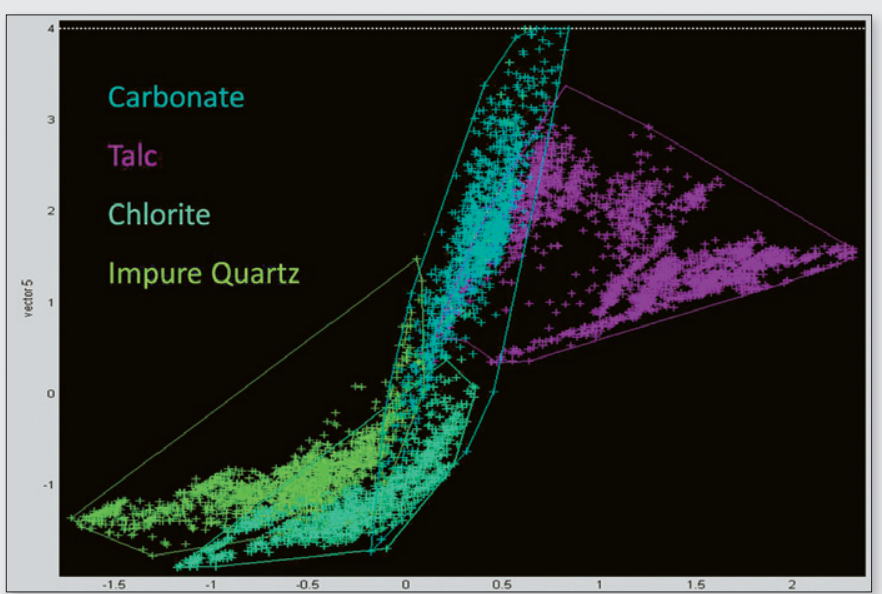

Figure 4. Classifier built up for the sorting test on the TITECH autosort. ${ }^{4}$
1000-2632 nm range. Ten scans were collected and averaged for each sample after which spectra were analysed, compared and checked for repeatability. The result of this evaluation is a representative group of spectra per rock type (Figure 3). Quartz is not infrared active but the spectra of impure quartz are dominated by absorptions associated with other components, like water and clay minerals.

It can be concluded that all four lithologies are causing diagnostic absorption features in the NIR. The measured absorption features are strong, diagnostic and repeatable. The repeatability is also confirmed because the spectra of the talc, chlorite and carbonate samples are comparable with the spectra found in the literature. Due to this, it can be expected that the separation is feasible for other deposits with the same lithologies. Separation based on NIR spectroscopy for talc is theoretically possible.

\section{Trials on a NIR sorter}

The NIR sorters of CommodasUltrasort are able to detect the characteristic NIR wavelength reflected by illuminated objects at two wavelengths between $800 \mathrm{~nm}$ and $1800 \mathrm{~nm}$. The sorter first needs to be trained with pre-classified (robust and clearly identified) samples. Groups are formed by statistical means and applied to the sorting algorithm, referred to as the classifier (Figure 4). The classification result can be improved by various image processing operations. Bulk tests with the TITECH autosort with a standard NIR scanner and with the Pro Secondary Color NIR combination of CommodasUltrasort (Figure 5) showed promising results. The sorted fractions had a particle size of

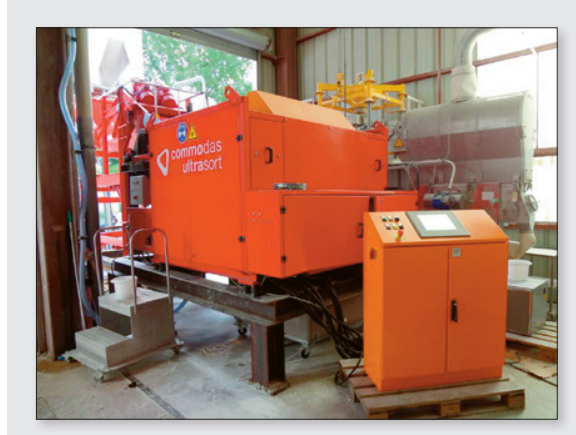

Figure 5. Pro Secondary Color NIR CommodasUltrasort sorting machine. Source: Reference 2.

20-40 mm and 40-60 mm while the sorting task was to get pure talc, which means increasing the whiteness and decreasing the carbonate content. Talc recovery after sorting was around $91-87 \%$ with a very low waste content of $2 \%$. The talc content in the waste fraction was around $22-30 \%$ and the talc loss after sorting was between $9 \%$ and $13 \%$.

\section{Conclusions}

This article describes successful applications of NIR spectroscopy in the minerals industry which highlights its potential and versatility. NIR spectroscopy can be used to produce mineralogy measurements at a frequency that would otherwise not be practical or affordable. Therefore, analysing the mineral composition of dry bulk materials using NIR-based on-line technologies is an emerging market. The remote sensing application is already common in the mining industry but still growing. While being feasible for many applications already today, it is expected that this technique will play an even more important role in the minerals industry in the future. NIR sorting is already state-of-the-art and is already widely implemented in the recycling and food industry. The first sorters in the minerals industry are already implemented (borate, limestone etc.) and it has proven its operational stability in, for example, the talc industry. This and extended pilot test work show the potential of the NIR sorting in the minerals industry.

\section{References}

1. P. Flinn, "An average day (or how near infrared affects daily life)", NIR news 16(17), 4 (2005).

2. M.R. Robben, C. Korsten, N. Pressler and P.-L. Audy, Theory and Operational Experience of NIR Sorting in the Talc Industry. Conerence Sensor Based Sorting, Aachen, Germany (2012)

3. R.N. Clark, "Spectroscopy of rocks and minerals, and principles of spectroscopy", in Manual of Remote Sensing, Vol. 3, Ed by A.N. Rencz and R.A. Ryerson. Wiley, pp. 3-58 (1999).

4. F. Sabins, Remote Sensing, Principles and Interpretation. W.H. Freeman and Company, New York (1997).

5. F. Riedel and H. Wotruba, "Review of sorting technologies", AMIRA Project P902: Dry Processing Review, Ch. 5. RWTH Aachen (2005).

6. M. Robben, M. Buxton, W. Dalmijn, H. Wotruba and D. Balthasar, "NIRS sorting in the upgrading and processing of Skorpion non-sulfide zinc ore", in Proceedings XXV International Mineral Processing Congress, Australia, Brisbane (2010). 